\title{
Sjogren's Syndrome Presenting with Distal Renal Tubular Acidosis in Adolescence
}

\author{
Zephyr D Dworsky ${ }^{1}$, Christine Bakhoum², Peter Chiraseveenuprapund ${ }^{3}$, Manaswitha Khare ${ }^{2}$ and \\ Noureddin D Nourbakhsh ${ }^{2 *}$ \\ ${ }^{1}$ Department of Pediatrics, Division of Hospital Medicine, UCSD Medical Center/Rady Children's Hospital, San Diego, CA \\ ${ }^{2}$ Department of Pediatrics, Division of Nephrology, UCSD Medical Center/Rady Children's Hospital, San Diego, CA
}

${ }^{3}$ Department of Pediatrics, Division of Rheumatology, UCSD Medical Center/Rady Children's Hospital, San Diego, CA

*Corresponding author: Noureddin Dean Nourbakhsh, Assistant Clinical Professor, Department of Pediatrics, Division of Nephrology, UCSD Medical Center/Rady Children's Hospital, San Diego, CA

\section{ARTICLE INFO}

Received: 慧 August 02, 2019

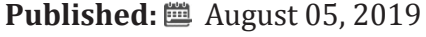

Citation: Zephyr D Dworsky, Christine Bakhoum, Peter Chiraseveenuprapund, Manaswitha K, Noureddin D Nourbakhsh. Sjogren's Syndrome Presenting with Distal Renal Tubular Acidosis in Adolescence. Biomed J Sci \& Tech Res 20(2)-2019. BJSTR. MS.ID.003433.

Abbreviations: SS: Sjogrens Syndrome; TIN: Tubulointerstitial Nephritis; ABPM: Ambulatory Blood Pressure Monitor; UVJ: Ureteric/Ureterovesical Junction; EGD: Esophago Gastro Duodenoscopy; AKI: Acute Kidney Injury

\begin{abstract}
Sjögreńs syndrome (SS) is a chronic progressive autoimmune disease primarily involving exocrine glands with varying degrees of systemic involvement, which can include severe interstitial lung disease, cutaneous vasculitis, peripheral neuropathy, and hematologic complications such as lymphoma. SS may also be associated with QT prolongation. Tubulointerstitial nephritis (TIN) may be a manifestation of renal involvement in primary SS in adults. Primary SS is isolated, whereas secondary SS is associated with other autoimmune diseases, such as rheumatoid arthritis, systemic lupus erythematosus, or scleroderma. Primary SS predominantly affects middle-aged women and is uncommon in childhood, with clinical manifestations differing between children/ adolescents when compared to adults.

In this case series, we review the clinical presentation of two adolescent patients whose diagnosis of Sjogren's syndrome and distal renal tubular acidosis (RTA) were discovered concurrently. Patient A is a 17-year-old female without classic sicca symptoms whose distal RTA served as the first indicator of SS disease. Patient B is a 12-year-old female who developed a distal RTA two months following prednisone taper while admitted for treatment of autoimmune hepatitis. In children, primary SS may be under-diagnosed because of the lack of classic sicca symptoms. However, SS should be included in the differential diagnosis of distal RTA and systemic symptoms, as prompt clinical diagnosis and treatment may lead to resolution of symptoms.
\end{abstract}

\section{Clinical Presentation}

\section{Patient A}

A 17-year-old female with a history of bilateral sensorineural hearing loss developed symptoms of intermittent fevers, body aches, headaches, and weakness 1-2 months prior to admission. She recalled a sensation of her "clothes feeling like they were stinging her body". Two months prior to admission, the working diagnosis was a viral illness. One month prior to admission, she experienced significant weight loss (17 lbs.), decreased appetite and nearly daily fevers. She also recalls feeling worsened muscle aches until the point of being unable to walk without assistance 1 week prior to admission. Dyspnea, both at rest and with exertion, increased 1 month prior to admission and were made worse when in a cold environment. She was evaluated in the Emergency Department for pulmonary embolus and CT angiogram chest was normal. A serum chemistry was not performed at the time, but urinalysis did show a $\mathrm{pH}$ of 7 , proteinuria and microscopic hematuria. She was then discharged home.

A few days later, blood work was performed at her primary pediatrician's office, prompting admission for severe hypokalemia and a metabolic acidosis. Upon admission, laboratory findings included elevated ESR, non-anion gap metabolic acidosis (primary process with respiratory compensation), hypokalemia, normocytic anemia, absence of urinary acidification ( $\mathrm{pH} \mathrm{7)}$ and nephrocalcinosis (Table 1). Renal ultrasound revealed echogenic kidneys with punctate, echogenic non-shadowing foci in collecting system bilaterally, suggestive of nephrocalcinosis (Figure 1). 
Table 1: Lab findings.

\begin{tabular}{|c|c|c|}
\hline Laboratory Findings & Patient A & Patient B \\
\hline Hospital Day (of RTA diagnosis) & 1 & 9 \\
\hline Serum $\mathrm{CO}_{2}(\mathrm{mmol} / \mathrm{L})$ & 9 & 14 \\
\hline Serum Potassium (mmol/L) & 2.5 & 2.2 \\
\hline Serum Chloride (mmol/L) & 122 & 123 \\
\hline Anion Gap (mmol/L) & 7 & 9 \\
\hline Urinary $\mathrm{pH}$ & 7 & 7.5 \\
\hline Urine Anion Gap & 17.6 & 3.5 \\
\hline TTKG (Transtubular Potassium Gradient) & 8.41 & Not evaluated \\
\hline $\begin{array}{c}\text { Serum Cr } \\
\text { eGFR (Schwartz I) }\end{array}$ & $\begin{array}{c}0.99 \mathrm{mg} / \mathrm{dL} \\
92 \mathrm{~mL} / \mathrm{min} / 1.73 \mathrm{~m}^{2}\end{array}$ & $\begin{array}{c}0.69 \mathrm{mg} / \mathrm{dL} \\
114 \mathrm{~mL} / \mathrm{min} / 1.73 \mathrm{~m}^{2}\end{array}$ \\
\hline Nephrolithiasis & Yes & Yes \\
\hline SS-A Antibody & $>8$ & $>8$ \\
\hline SS-B Antibody & $>8$ & $>8$ \\
\hline Salivary Gland Biopsy Results & $\begin{array}{l}\text { Scattered small foci of lymphoplasmacytic } \\
\text { infiltrates. } \\
\text { No significant acute inflammation is seen. } \\
\text { Granulomas are not identified. } \\
\text { Lymphoepithelial lesions are not seen. }\end{array}$ & $\begin{array}{c}\text { Moderately dense infiltrate of largely periductal lympho- } \\
\text { cytes and plasma cells, with a focal suggestion of early } \\
\text { secondary germinal center formation. } \\
\text { The focus score of lymphocytic inflammation per } 4 \mathrm{~mm} \\
\text { squared cross-section is at least } 3 \text {. } \\
\text { No significant gland atrophy, fibrosis, or adipose replace- } \\
\text { ment. }\end{array}$ \\
\hline
\end{tabular}

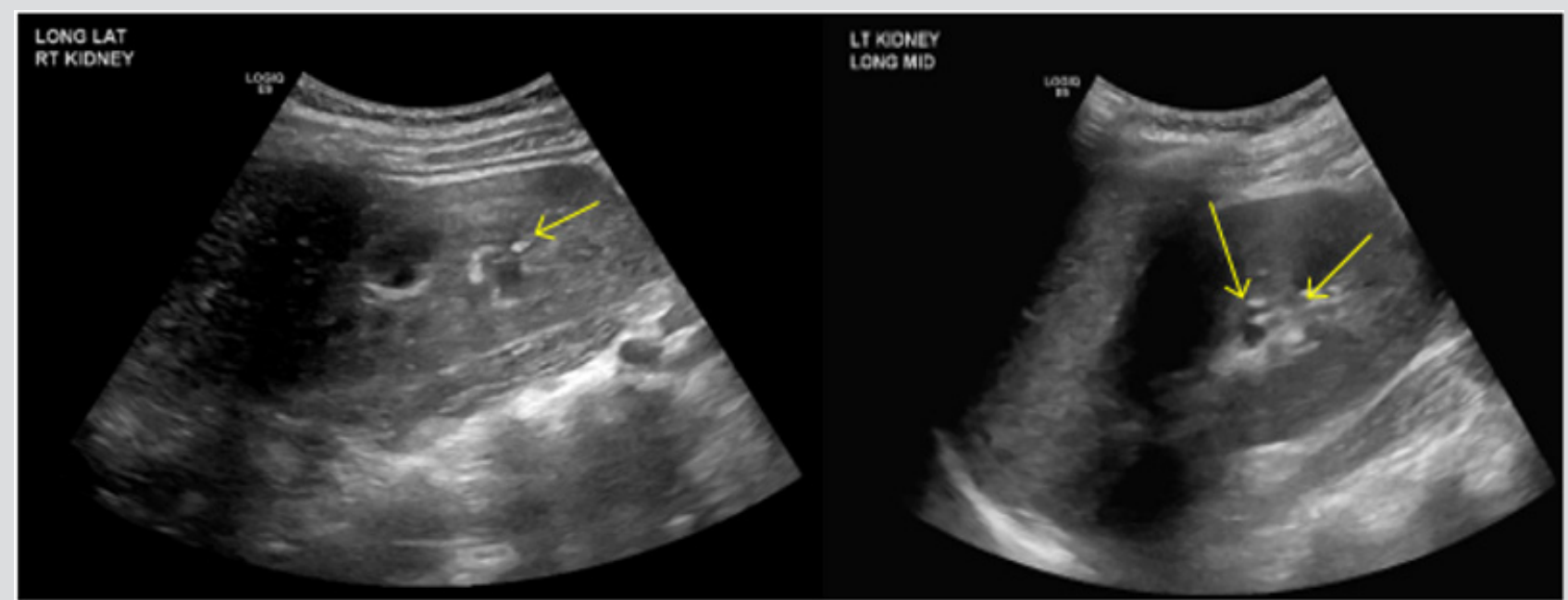

Figure 1: Nephrocalcinosis; Patient A - 1-2 mm echogenic focus in R renal collecting system (arrow). 1-2 mm echogenic foci (2) in $\mathrm{L}$ renal collecting system (2 arrows).

\section{Patient B}

A 12-year-old female was referred for evaluation by gastroenterology for elevated liver enzymes. Her liver enzymes had been elevated for three years, with AST of 150 and ALT of 165 at the time of presentation. Further work up by gastroenterology revealed ANA speckled pattern titer 1:1280 and nucleolar pattern titer 1:40 as well as elevated IgG level to 4,367. This prompted evaluation with CT guided liver biopsy which confirmed the diagnosis of autoimmune hepatitis. The patient was started on prednisone 60 mg daily and azathioprine. Patient was hospitalized twice in the month following the diagnosis for an increase in LFTs. During that time, she received IV solumedrol and was discharged again on oral prednisone. LFTs remained elevated and were refractory to both steroid therapy and azathioprine therapy. Patient was started on mycophenolate mofetil and azathioprine was discontinued. 
She was noted to be hypertensive and was referred for 24-hour ambulatory blood pressure monitor (ABPM). ABPM confirmed severe ambulatory hypertension while patient on prednisone 10 mg twice daily. She was admitted for further management and evaluation of her hypertension. During this admission, she was found to have bilateral sub-centimeter, non-obstructing renal stones on ultrasound. Urine calcium to creatinine ratio at that time was mildly elevated at 0.23 . She was started on hydrochlorothiazide in addition to metoprolol for management of her hypertension. The rest of her evaluation for hypertension included: normal kidney function with $\mathrm{Cr} 0.52 \mathrm{mg} / \mathrm{dl}$, normal C3, C4, negative dsDNA, normal urine protein to creatinine, normal renin and aldosterone, elevated plasma metanephrines but a negative MIBG scan. With the start of HCTZ, she required potassium chloride supplementation. Hypertension was well controlled, and she was discharged home.

Over the course of the next two months, prednisone was tapered off. Her hypertension subsequently improved and she was taken off of HCTZ and metoprolol. She then developed persistent nausea and refractory emesis with several episodes daily. She lost 14 lbs over the course of 6 weeks and was admitted again for further evaluation. During this admission, her initial electrolytes were remarkable for severe hypokalemia, non-anion gap metabolic acidosis, positive urine anion gap and urine $\mathrm{pH}$ of 7.5 (Table 1). CT abdomen and pelvis without contrast revealed bilateral nephrolithiasis with a left distal ureteric/ureterovesical junction (UVJ) calculus, resulting in mild left hydroureter (Figure 2).

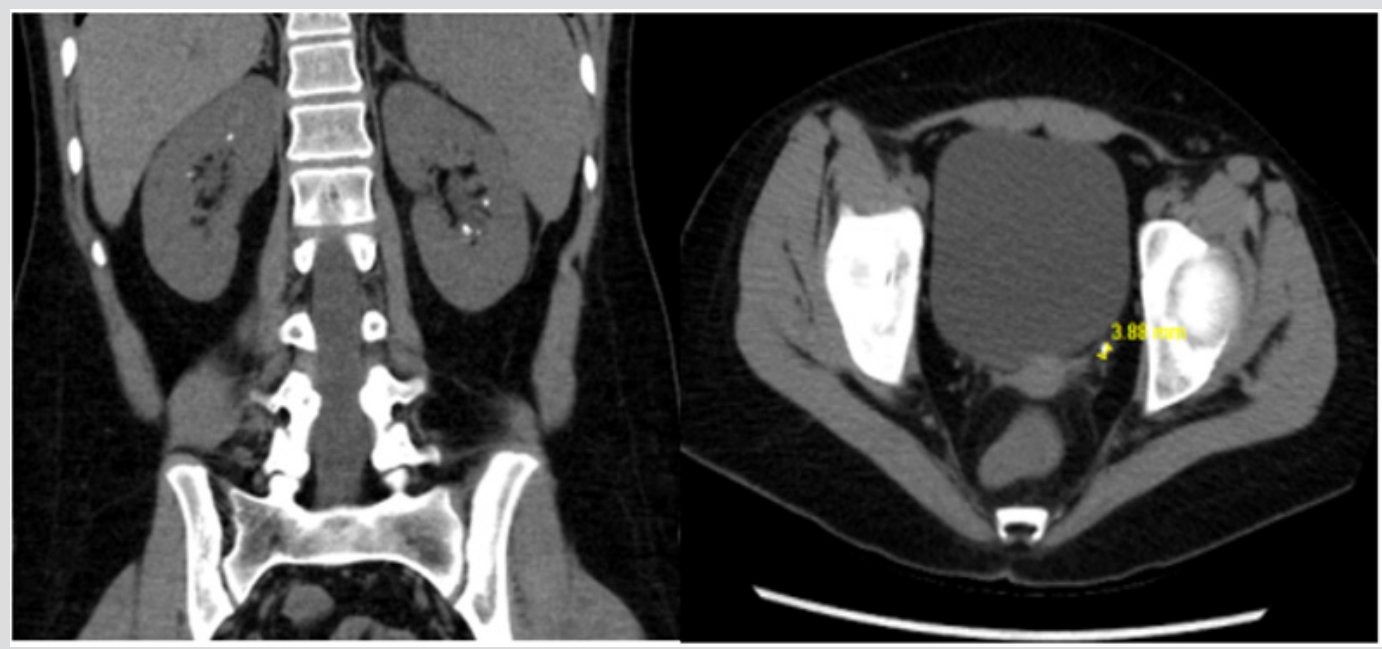

Figure 2: Nephrolithiasis; Patient B - Bilateral intrarenal collecting system calculi. Mild diffuse left hydroureter, there is a $4 \mathrm{~mm}$ calculus at the left ureterovesical junction, with mild periureteric stranding.

\section{Clinical Course}

\section{Patient A}

Patient A initially received parenteral electrolyte repletion with improvement in her electrolyte derangements. She underwent further work up for suspected rheumatologic condition, with suspicion for Sjogren's syndrome given its association with distal renal tubular acidosis in adults [1,2]. She was found to have significantly elevated ANA, SS-A and SS-B, elevated anti-smooth muscle antibody and amylase as well as total IgG and IgG1 levels. Further rheumatologic work up was negative, including normal complements, antiphospholipid antibodies, ANCA vasculitides antibody panel, dsDNA, anti-Smith antibody and RNP antibodies. Her coagulation panel and thyroid function studies were also normal. She underwent infectious work-up including blood cultures, respiratory viral panel, HIV and hepatitis C testing, all of which were negative. Her initial electrocardiogram showed a prolonged QTc that was followed and normalized with correction of her electrolyte abnormalities. She was found to have a normocytic anemia with hemoglobin of $9.3 \mathrm{~g} / \mathrm{dL}$ on admission associated with reticulocytosis and a positive fecal occult blood. After her electrolytes stabilized, she underwent a salivary gland biopsy and esophagogastroduodenoscopy/colonoscopy. The endoscopy was significant for several oozing gastric ulcers, but otherwise unremarkable. Her salivary gland biopsy showed lymphoplasma cytic infiltrates, which appeared to meet the proposed criteria for Sjogren's syndrome. Based on immunohistochemistry staining, it did not appear to be secondary to IgG4-releated disease or sarcoidosis. She was thus started on prednisone therapy and transitioned to enteral electrolyte repletion.

After initiation of prednisone, her symptoms began improving. She also required the addition of hydroxychloroquine and azathioprine. With immunosuppression, her fevers, myalgias and dyspnea resolved. Further work-up has revealed undetectable urinary citrate levels on 24-hour urine collection, for which she receives potassium citrate. Her elevated inflammatory markers have improved dramatically with most recent ESR only slightly above normal. Her anemia similarly has improved, though she 
remains slightly anemic. Currently, she is doing well and is in remission of disease while on prednisone, hydroxychloroquine and azathioprine.

\section{Patient B}

Patient B underwent further rheumatologic workup and was found to have significantly elevated Sjogren's Antibodies with SS-A and SS-B both > 8.0 (Table1). ANA remained positive with negative dsDNA. Anti-smooth muscle antibody, anti-Smith and RNP antibodies were negative. Thyroid antibodies were negative. Her IgG4 level was low at $<0.8$. Salivary gland biopsy showed a moderately dense infiltrate of periductal lymphocytes and plasma cells, consistent with Sjogren's syndrome. Sjogren's syndrome was thought to be the cause of her distal renal tubular acidosis.

Patient then underwent ureteroscopy with laser lithotripsy for the obstructive UVJ stone. She continued to require large amounts of electrolyte repletion. Following the procedure, she developed pancreatitis, with lipase level as high as 1,651. Eventually her symptoms improved and she was able to transition to oral supplementation with potassium citrate. Regarding treatment of her Sjogren's syndrome, she received one dose of rituximab $500 \mathrm{mg} / \mathrm{m}^{2}$ during her hospitalization with a plan for a second dose two weeks afterward. Rituximab was chosen as the primary therapy of choice based on the severity of her disease: autoimmune hepatitis, pancreatitis, and distal renal tubular acidosis, all sequelae of Sjogren's syndrome. Low dose prednisone was not initiated in conjunction with rituximab, as she did not seem to respond to steroids in the past and had severe adverse effects including hypertension and severe striae. Her electrolyte abnormalities have resolved on potassium citrate therapy.

\section{Discussion}

Sjogren's syndrome is a chronic, progressive autoimmune disorder that primarily involves the exocrine glands, including lacrimal and salivary glands. It is rare in children, with clinical manifestations differing in the pediatric population compared to adults. Children tend to present with more systemic symptoms rather than sicca symptoms, with renal involvement reported to range from $7.1-19.2 \%$ [3]. In this case series, we review the presentation of two adolescent females whose primary diagnosis of Sjogren's syndrome was uncovered coincident with, and in patient A's case as a result of, development of distal renal tubular acidosis. Although renal tubular findings associated with primary SS is an underappreciated manifestation in pediatric patients, there appears to be a significant morbidity associated with renal involvement in primary SS in adults. Goules et al. [4] studied 715 adult patients in a European cohort who met criteria for primary SS and found that $4.9 \%$ of patients had significant renal involvement.

In this cohort, overall 5-year survival rate was $85 \%$, but there was a statistically significant reduction in survival for patients with primary SS with renal involvement [4]. In most cases, primary
SS affects the renal tubules through TIN, in which infiltration of the kidney by plasma cells is a key feature similar to the lymphoplasmacytic infiltration of the salivary glands [5]. Although various segments of the nephron can be affected, distal renal tubular acidosis is the most common RTA appreciated in patients with primary SS, possibly related to decreased expression of $\mathrm{H+}$ ATPase and alpha-1 anion (AE-1) exchanger in the intercalated cells [6]. Clinically, patients can present with profound muscle weakness, periodic paralysis, nephrocalcinosis and osteomalacia. Prompt diagnosis of renal tubular dysfunction as either a sequela of primary SS or, as in our case series, an indicator of diagnosis of primary SS, is of vital importance in alleviating symptoms and minimizing renal dysfunction. A course of glucocorticoid therapy can reverse the biochemical lab abnormalities of distal RTA and may minimize the tubular atrophy and interstitial fibrosis associated with longterm renal tubular dysfunction [7]. While primary SS is known to be the most common acquired cause of distal RTA in adults, the differential diagnosis for distal RTA in pediatric literature does not typically include primary SS.

In our case series, both adolescent patients developed the classic clinical findings of distal RTA and diagnosis of primary SS was not only verified by SS-A/SS-B antibody testing, but also by salivary gland biopsy. Patient A presented in a near paralytic state, with dyspnea presumably secondary to electrolyte abnormalities (hypokalemia, hypophosphatemia) associated with distal RTA. Given her history of weight loss and other constitutional symptoms, the diagnosis of Sjogren's syndrome was sought and work-up was performed to that end. Once started on potassium and citrate supplementation, her dyspnea, muscle weakness and QT prolongation improved. Furthermore, following initiation of prednisone therapy, reduced doses of potassium and citrate were required to maintain normal biochemical profile. In the case of Patient B, primary diagnosis was autoimmune hepatitis and the diagnosis of primary SS was only uncovered during an admission for weight loss and electrolyte abnormalities associated with distal RTA. We postulate that patient B's distal renal tubular acidosis was not present at time of initial diagnosis of autoimmune hepatitis due to prolonged therapy with glucorticoids. Her distal RTA may have only come to clinical attention following discontinuation of glucocorticoid therapy. Given patient B's history of steroid toxicity, resumption of glucocorticoid therapy was deferred and primary immunosuppression with rituximab was implemented. Both patients were found to have nephrocalcinosis, and in patient B's case, an obstructing left uterovesiculojunction (UVJ) calculus, led to acute kidney injury (AKI). AKI resolved following lithotripsy and her RTA was treated with citrate therapy.

While in the adult literature distal RTA as a result of tubulointerstitial nephritis associated with primary SS is well described, there is a paucity of pediatric literature connecting renal tubular dysfunction and primary SS. In this case series, we 
review the clinical presentation of two adolescent patients whose diagnosis of Sjogren's syndrome and distal RTA were discovered concurrently and therefore, treated promptly. In children, primary SS is an exceedingly rare diagnosis and the lack of classic sicca symptoms in children with primary SS makes the diagnosis more challenging. However, SS should be included in the differential diagnosis of distal RTA and systemic symptoms, as prompt clinical diagnosis and treatment may lead to resolution of symptoms.

\section{References}

1. Jung SW, Park EJ, Kim JS, TW Lee, Chun GI, et al. (2017) Renal Tubular Acidosis in Patients with Primary Sjögren's Syndrome. Electrolyte Blood Press 15(1): 17-22.

2. Sarah S, Lijo G, Sukanya E, Rajasekaran D (2015) Renal tubular acidosis

ISSN: 2574-1241

DOI: $10.26717 /$ BJSTR.2019.20.003433

Noureddin Dean Nourbakhsh. Biomed J Sci \& Tech Res

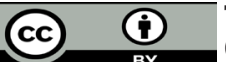

This work is licensed under Creative

Commons Attribution 4.0 License

Submission Link: https://biomedres.us/submit-manuscript.php due to Sjogren's syndrome presenting as hypokalemic quadriparesis: A report of two cases. Indian J Nephrol 25(6): 386-387.

3. Virdee S, Greenan Barrett J, Ciurtin C (2017) A systematic review of primary Sjögren's syndrome in male and paediatric populations. Clin Rheumatol 36(10): 2225-2236.

4. Goules AV, Tatouli IP, Moutsopoulos HM, Tzioufas AG (2013) Clinically significant renal involvement in primary Sjogren's syndrome: clinical presentation and outcome. Arhtritis Rheum 65(11): 2945-2953.

5. Francois H, Mariette X (2016) Renal involvement in primary Sjogren syndrome. Nat Rev Nephrol 12(2): 82-93.

6. Kim HY, Kim SS, Bae EH, Ma SK, Kim SW (2015) Decreased Renal Expression of H (+)-ATPase and Pendrin in a Patient with Distal Renal Tubular Acidosis Associated with Sjogren's Syndrome. Intern Med 54(22): 2899-2904.

7. Maripuri S, Grande JP, Osborn TG, Fervenza FC, Matteson EL, et al. (2009)

$\begin{array}{ll}\text { BIOMEDICAL } & \text { Assets of Publishing with us } \\ \text { RESEARCHES } & \text { - Global archiving of articles } \\ & \text { - Immediate, unrestricted online access } \\ & \text { - Rigorous Peer Review Process } \\ \end{array}$

